\title{
El legado de la urbanización europea en el cono sur sudamericano: Una aproximación a la historia de las teleconexiones sobre la ecología del territorio
}

\author{
H. Ricardo Grau ${ }^{12, \otimes}$ \& Javier Foguet ${ }^{1}$ \\ ${ }^{1}$ Instituto de Ecología Regional (Universidad Nacional de Tucumán - CONICET). Yerba Buena, Tucumán, Argentina. \\ ${ }^{2}$ Facultad de Ciencias Naturales, Universidad Nacional de Tucumán.
}

\begin{abstract}
Resumen. Analizamos la potencial influencia histórica de las ciudades fundadas a partir de la colonización europea sobre el territorio del cono sur sudamericano. Para ello describimos los patrones temporales de fundación de ciudades, elaboramos mapas del área influencia potencial de núcleos urbanos en base a su fecha de fundación y caracterizamos las ecorregiones de acuerdo con su historia de influencia urbana. En Chile, Paraguay y Argentina, las ciudades se fundaron mayormente antes de 1650 (inicio de la colonización española) y posteriormente a 1850 (durante la consolidación de los estados nacionales). En Uruguay y en el sur de Brasil, la colonización ocurrió principalmente entre 1650 y1850. El patrón bimodal del sector oeste se refleja también en las ecorregiones del Monte, el Chaco Semiárido y el Chaco Húmedo. El centro-oeste y noroeste del área de estudio incluye ecorregiones ambientalmente muy distintas (la Puna, las Yungas y el Matorral chileno), pero con una historia común de influencia temprana de ciudades fundadas en el siglo XVI. En contraste, las ecorregiones de la Patagonia muestran una escasa y tardía influencia urbana sobre el territorio a partir de fines del siglo XIX. Las ecorregiones del este, las Pampas y el Bosque Atlántico se caracterizan por fechas intermedias de colonización. Entre los procesos ecológicos potencialmente asociados a estos patrones se destacan: 1) la historia de agricultura y, principalmente, ganadería, y sus potenciales efectos ecológicos, 2) las perturbaciones asociadas a la colonización, temporalmente discretas, pero con efectos ecológicos duraderos (e.g., pulsos de fuego y epidemias), 3) el efecto retardado de la fundación temprana como condicionante del tamaño urbano actual, que, a su vez, influye sobre las transiciones ecológicas y la invasión de especies, y 4) la aparición de 'nichos socioecológicos' distintivos en las zonas de colonización tardía (e.g., los menonitas en el Chaco). Este estudio brinda una visión integradora y metodológicamente promisoria para analizar la historia ecológica de las teleconexiones, clave del funcionamiento del Antropoceno.
\end{abstract}

[Palabras clave: Antropoceno, ciudades, colonización, ecorregiones, globalización, historia ambiental]

Aвstract. The legacy of European urbanization in southern cone of South America: An approach for the study of the history of teleconnections on territorial ecology. We analyzed the potential historical influence of cities in the South American Southern Cone. To do so, we described temporal patterns of cities' foundations; we built maps of potential influence of urban centers and characterized ecoregions according to their history of 'influential' cities. In Chile, Paraguay and Argentina, cities were mostly founded prior to 1650 (early Spanish colonization) and after 1850 (consolidation of national states). In Uruguay and Brazil, colonization occurred mostly between 1650 and 1850 . The bimodal pattern observed in the western side of the study area is also reflected in the Monte, Arid Chaco and Humid Chaco ecoregions. The center-west and northwest of the area include ecoregions which are environmentally very different (Puna, Yungas, Chilean matorral), but have a common early history of cities' influence during the $16^{\text {th }}$ century. In contrast, the ecoregions of the Patagonia show low and late urban influence, starting in the $19^{\text {th }}$ century. The ecoregions of the east (Pampas and Atlantic forest) are characterized by intermediate colonization dates. Among the ecological processes potentially associated with these patterns of cities foundation, we identify the following: 1) history of agriculture and, most importantly, livestock herbivory and its ecological consequences, 2) short term perturbations with longlasting effects (e.g., pulses of fire activity, human diseases), 3) the delayed effect of early cities foundations as cause of current urban size, which in turns influences ecological transitions and exotic species invasions, and 4) the emergence of novel 'social-ecological' niches in areas of late colonization (e.g., Mennonites in the Chaco). This study provides an integrative and promising methodological approach to analyze the ecological history of land-based teleconnections, a key for the functioning of the Anthropocene.

[Palabras clave: Anthropocene, cities, colonization, ecoregions, globalization, environmental history, environmental history]

Editor asociado: Germán Baldi
Recibido: 11 de Agosto de 2020

Aceptado: 5 de Noviembre de 2020 


\section{INTRODUCCIÓN}

Los patrones históricos de uso del territorio juegan un papel central en la ecología del Antropoceno (Verburg et al. 2015). Los intentos de mapear la actividad humana en el pasado se enfocaron en modelar el 'impacto' humano, entendido como acciones que transforman los ecosistemas naturales mediante actividades locales de caza, recolección y, principalmente, agricultura y ganadería de distintas intensidades. Los esfuerzos más sistemáticos en este sentido (e.g., 'Antromas') (Ellis and Ramankutty 2008; Ellis et al. 2013, 2020) se basan sobre estimaciones de la densidad de la población humana y los tipos genéricos de usos del territorio (e.g., residencial, agrícola, ganadero), actividades que por lo general resultan en reducción de la biomasa y biodiversidad de los ecosistemas. Además, en décadas recientes se documentó que la influencia humana sobre el territorio puede ir en la dirección opuesta - de expansión de bosques $\mathrm{u}$ otros ecosistemas naturales-, ya sea por acciones deliberadas de restauración ecológica o por des-intensificación del uso resultante de las dinámicas demográficas y socioeconómicas (e.g., Grau and Aide 2008; Lambin and Meyfroidt 2010).

Estos análisis históricos se enfocan en el uso local del territorio, sin referencia explícita a los procesos derivado de las conexiones distantes con otras regiones. A estas 'teleconexiones' y 'teleacoples', sin embargo, se las reconoce cada vez más como aspectos centrales del funcionamiento socioecológico al vincularse, por ejemplo, con la apertura a distintos mercados o al incorporar patrones culturales originados en nuevos grupos sociales que pasan a habitar o influir sobre una región (Friis et al. 2017). En las américas, por ejemplo, un hito crucial en la historia socioecológica es la llegada y colonización Europea a partir de 1492 (Crosby 1993; Mann 2005; Brailovsky 2006). Antes, el continente incluía una variedad de culturas agrícolas sedentarias asociadas a infraestructura y estructuras gubernamentales sofisticadas, en una matriz de territorio ocupado por grupos agrícolas menos desarrollados y grupos nómades de cazadores-recolectores. A partir del siglo XVI, la población y la cultura local fue reducida drásticamente y gradualmente mestizada o reemplazada por otra con un uso del territorio muy distinto (Williamson 2009). Adicionalmente, este evento implicó que tras haber estado aislada al menos durante todo el Holoceno, la biota americana se re-conectara con el resto del mundo y reorientara su funcionamiento socioecológico a la nueva realidad política de dependencia de Europa y a las crecientes conexiones con el viejo mundo (Mann 2013). Las nuevas tecnologías (incluyendo en las etapas iniciales un fuerte impacto de la minería), formas de tenencia de la tierra y especies que se establecieron son las que, en buena medida, definen el paisaje actual. Estas incluyen, por ejemplo, nuevos cultivos (e.g., trigo, caña de azúcar, vid, cítricos, frutales de pepita y carozo), nuevos herbívoros (e.g., vacunos, ovinos, porcinos, caprinos y equinos), nuevos medios de transporte (e.g., la rueda, las embarcaciones, bueyes, caballos, burros y mulas; más tarde, motores a explosión), nuevas formas de trabajar el suelo (e.g., arados con tracción y siembra mecanizada), tenencia de la tierra basadas inicialmente en reducciones y encomiendas, seguidas por emprendimientos capitalistas, y una estructura de poder político centralizado y estrechamente asociado a la religión católica. Como elemento distintivo, a la vez causa y efecto de la nueva configuración del territorio, emergieron las modernas ciudades.

Sudamérica es la región del planeta donde la población urbana representa la mayor proporción de la población total: más del $80 \%$. Al albergar el grueso de la población, las ciudades concentran la actividad comercial, las instituciones y las decisiones sobre el uso del territorio, incluyendo tanto procesos de expansión e intensificación del uso del territorio como procesos de des-intensificación agrícola y 'transición forestal' (Grau and Aide 2008; Grau 2016). El grueso de las ciudades latinoamericanas actuales se estableció a partir de la colonización europea, generalmente con una fundación formal en una fecha precisa y una localización funcional a centros distantes de poder político-económico (Williamson 2009). Estas dos características, fecha confiable de establecimiento y rol central en el desarrollo regional 'globalizado', convierten a las ciudades sudamericanas en un valioso recurso para describir los patrones espaciotemporales de historia de uso del territorio, en particular, en lo referido a su integración a la economía, política y ecología global.

El cono sur de Sudamérica, definido en forma general como el territorio situado al sur del trópico de Capricornio, presenta buenas oportunidades para evaluar el potencial de las ciudades como aproximación a la historia moderna de uso del territorio. Incluye cinco países, que a su vez reflejan la mayor 
divergencia en la historia temprana colonial de Sudamérica: el Tratado de Tordesillas (1494), que dividió el territorio entre dos potencias imperiales de la época, España y Portugal, lo que posteriormente resultó en procesos de independización distintos. Incluye tanto las zonas representativas de la agricultura precolonial más desarrollada (Tahuantinsuyo, Araucanía/Wallmapu) como zonas extensas que al momento de la colonización estaban ocupadas por grupos cazadores-recolectores o agricultores de menor escala. Comprende un gradiente ambiental extenso que incluye desiertos extremos (menos de $10 \mathrm{~mm}$ de lluvias anuales), selvas lluviosas subtropicales o templadas con varios metros de precipitación anual, y zonas frías de alta montaña o altas latitudes (Cabrera and Willink 1980).

El objetivo general de este estudio es modelar espacialmente la potencial influencia socioecológica de las ciudades coloniales y post-coloniales sobre el territorio en el cono sur sudamericano. Para esto, nos enfocamos en tres objetivos específicos: (1) describir los patrones espacio-temporales de las ciudades dominantes o 'influyentes' sobre el territorio, (2) elaborar mapas de influencia histórica potencial de núcleos urbanos, tipificados de acuerdo a su fecha de fundación, y (3) categorizar las ecorregiones del cono sur de acuerdo a su historia de influencia urbana histórica.

El modelado de la 'influencia potencial' de las ciudades aquí considerado simplemente supone que aquélla está controlada de manera directa por la distancia a éstas como base para posteriores modelos más sofisticados que deberían capturar las complejidades socioecológicas de los sistemas y explicitar las variables que definen la 'influencia'. En esta primera contribución a este objetivo, ejemplificamos potenciales efectos ecológicos de los patrones observados y discutimos la potencial aplicabilidad de este enfoque metodológico al estudio de la historia de uso del territorio 'teleconectado' en otras regiones.

\section{Materiales y Métodos}

Para describir los patrones espacio temporales de influencia histórica de las ciudades del cono sur (objetivo 1) tomamos como referencia jurisdicciones políticas de segundo orden que en líneas generales se sitúan al sur del extremo norte de la Argentina (paralelo $22^{\circ}$
S). Esto incluye la totalidad de las provincias argentinas (excluyendo la Antártida y las islas del Atlántico Sur), todos los departamentos de Uruguay, casi todas las regiones de Chile (excepto la $1^{a}$ [Tarapacá]), casi todos los departamentos de Paraguay (con la excepción del departamento de Alto Paraguay) y los tres estados más australes de Brasil (i.e., Paraná, Santa Catarina y Rio Grande do Sul). En conjunto, el área ocupa $4510604 \mathrm{~km}^{2}$.

Registramos el año de fundación de todas las ciudades del área de estudio que cumplieran con al menos una de las siguientes condiciones: 1) más 300000 habitantes y estar situada a más de $10 \mathrm{~km}$ de la 'mancha urbana' de una ciudad mayor, 2) más de 100000 habitantes, siempre que fueran la mayor ciudad en 100 $\mathrm{km}$ a la redonda, 3) más de 10000 habitantes, siempre que fueran la mayor ciudad en $200 \mathrm{~km}$ a la redonda, y 4) ser la capital de jurisdicción de segundo orden siempre que sea la ciudad más antigua en 200 km a la redonda. El punto (1) indica que no se consideraron independientemente ciudades cuya 'mancha urbana' (territorio cubierto por infraestructura) fuera espacialmente continua con ciudades mayores; es decir que en caso de ciudades adyacentes, se las consideró como parte de una sola entidad centrada en la de fecha de fundación más temprana. Las ciudades de Paraná (Argentina) y Ciudad del Este (Paraguay) se excluyeron del análisis por estar situadas a poca distancia de ciudades preexistentes por muchos años (Santa Fe y Caaguazú, respectivamente), lo que claramente representaba un uso del territorio bajo dominio europeo previo a la fundación de estas ciudades. En unos pocos casos (Curuzú Cuatiá [Argentina]; Umaurana y Guarapuava [Brasil]) no se consideró la fecha de fundación de ciudades sino la de establecimientos agrícola-ganaderos preexistentes en la zona, que claramente representaban la primera actividad económica vinculada con el dominio europeo. Estos criterios fueron considerados de manera independiente para cada país, es decir que en las distancias que definen los criterios (2), (3) y (4) no se consideró la distancia a ciudades de países vecinos. La población total considerada fue la de la totalidad del conglomerado urbano, entendiendo como tal aquellas estructuras urbanas que guardan continuidad espacial (e.g., el Área Metropolitana de Buenos Aires, incluyendo La Plata). Si bien se entiende que La Plata consistió en un evento fundacional independiente, este análisis tiene por objetivo 
determinar la influencia global sobre el territorio, algo que ya estaba claramente establecido por siglos en el momento de esta fundación. En todos los casos, los niveles poblacionales considerados fueron los de los censos o estimaciones más recientes reportados en Wikipedia (www.wikipedia.com).

Para determinar la fecha de fundación se usó como fuente primaria Wikipedia (versiones de Wikipedia en portugués para Brasil y en español para el resto de los países), complementada con búsquedas más detalladas en fuentes bibliográficas de internet cuando la fecha no era explicitada inicialmente. Esta plataforma resultó ser exactamente coherente con todas las fechas que figuraban en otra bibliografía utilizada (Piossek Prebisch 2001; Brailowsky 2006; Williamson 2009) y, para el nivel de resolución temporal considerado en el modelado espacial (100 años), resultó en una relación óptima entre el esfuerzo de obtención de los datos y la calidad del mapa generado. Dado que el objetivo de este trabajo es modelar la potencial influencia de la colonización europea, en todos los casos se consideró como fecha de fundación la generada por la autoridad colonial o nacional, desestimando los establecimientos urbanos o cuasi-urbanos indígenas preexistentes en la misma localización, lo que ocurrió en al menos dos casos (Copiapó y Calama, Chile). En el caso de ciudades fundadas en una localización, pero posteriormente trasladadas a una nueva, se consideró como fecha de fundación la correspondiente al asentamiento original, siempre que luego se hubieran trasladado a menos de $100 \mathrm{~km}$ de distancia; mientras tanto, para el modelado espacial se consideró la localización presente. Bajo los criterios considerados, en general se pudo establecer la fecha de fundación con precisión aproximadamente anual. Esto no se cumplió en dos casos, Foz do Iguazú (Paraná, Brasil) y Caxias do Sul (Rio Grande do Sul, Brasil), ciudades que se generaron de manera gradual e informal. En estos casos, la fecha propuesta fue estimada en base a los registros históricos aproximados referidos en Wikipedia o en las fuentes allí citadas. No se incluyeron ciudades fundadas por los europeos y después abandonadas, en general por efecto de ataques indígenas. Este es el caso, por ejemplo, del Fuerte Sancti Spiritu (Santa Fe, Argentina), Londres (Catamarca, Argentina), El Barco (Tucumán, Argentina), Rosario de Calchaquí (Salta, Argentina) o Villarica (Araucania, Chile). Consideramos que la destrucción temprana de estas ciudades implicó que el efecto de 'globalización' que se intenta modelar en este estudio no fue tal. Por otra parte, la fundación de ciudades cercanas (Santa Fe, San Fernando del Valle de Catamarca, San Miguel de Tucumán, Salta, Valdivia, respectivamente), con fechas posteriores, pero similares en relación con los lapsos temporales aquí tratados, hace que estas omisiones tengan relativamente poco peso sobre los patrones emergentes del modelado geográfico. Para visualizar estos resultados presentamos las fechas de fundación en cuatro categorías: hasta 1650, 1651-1750, 1751-1850, posterior a 1851.

Para modelar espacialmente la potencial influencia de cada ciudad (objetivo 2), mapeamos en un contexto de sistema de información geográfica (SIG) raster, la fecha de establecimiento de la ciudad más cercana a cada pixel. A partir de la coordenada del centroide de cada ciudad generamos polígonos de Voronoi, y a cada uno de ellos le asignamos como atributo la fecha de fundación de su ciudad de referencia (la ciudad más cercana a cada punto del territorio).

Para describir el patrón histórico de influencia urbana de cada ecorregión (objetivo $3)$, en primera instancia 'intersectamos' los modelos espaciales antes generados con las ecorregiones 'nivel 2' de World Wildlife Fund (http://ecologicalregions.info/htm/ sa_eco.htm). Para el total del área de estudio se incluyeron 12 ecorregiones: Fiordos y bosques fueguinos (Chile, Argentina), Estepa Patagónica (Argentina, Chile), Bosques Valdivianos (Chile, Argentina), Monte (Argentina), Matorral Mediterráneo chileno (Chile), Chaco Semiárido (Argentina, Paraguay), Chaco Húmedo (Argentina, Paraguay), Pampa Austral (Argentina), Pampa Ondulada Septentrional (Uruguay, Brasil), Bosque Atlántico (Brasil, Paraguay y Argentina), Puna-Altos Andes (Argentina, Chile) y Yungas Australes (Argentina). Aunque se incluían en el área de análisis, no consideramos para el objetivo 3 las ecorregiones Desierto Atacameño (Chile) y Cerrado (Brasil) debido a su superficie menor en el área de estudio, en comparación con el área total de estas ecorregiones. Se utilizó esta clasificación de ecorregiones porque si bien los distintos países tienen esquemas más refinados y precisos (e.g., discriminando las ecorregiones de Espinal y Esteros del Iberá en la Argentina), no son completamente consistentes entre países, lo que dificultaría 
el análisis trasnacional. Cada una de las 12 ecorregiones consideradas fue caracterizada en base al porcentaje de su superficie que caía en la zona de influencia de ciudades en las cuatro clases temporales de referencia. Para visualizar estos resultados, agrupamos las ecorregiones en cuatro patrones históricos definidos de forma visual.

\section{Resultados}

\section{Patrones temporales}

Bajo los criterios especificados, se determinó la fecha de fundación de un total de 99 ciudades de Chile (15), Paraguay (11), la Argentina (46), Uruguay (6) y Brasil (21) (ver Material Suplementario). La fecha de fundación de ciudades refleja dos patrones diferenciados (Figura 1a). Chile, Paraguay y la Argentina (sector oeste del área de estudio) se caracteriza por un patrón bimodal, con el mayor número de ciudades fundadas antes de 1650 bajo dominio de España, relativamente pocas entre 1650 y 1850, y un nuevo 'pulso' de fundaciones posterior a 1850, cuando estos territorios ya pertenecían a los estados independientes actuales. En este sector, el grueso de la población urbana se localiza actualmente en las ciudades más antiguas correspondientes a la primera ola de fundaciones (Figuras 1a, 2a). Las ciudades fundadas antes de 1650 hoy albergan $~ 80 \%$ de la población urbana incluida en este análisis e incluye las capitales (Santiago de Chile, Asunción y Buenos Aires), y la mayoría de las ciudades mayores a 500000 habitantes, con pocas excepciones (e.g., Rosario, Argentina, fundada en 1786).

En contraste, el sector este del cono sur (Uruguay y el sector sur de Brasil, con influencia portuguesa durante la colonización) registra sólo dos ciudades menores cuyos asentamientos se iniciaron antes de 1650 (Figuras 1a, 2b). En general, las ciudades comenzaron a establecerse cuando ya había finalizado el primer pulso de fundaciones en (a)

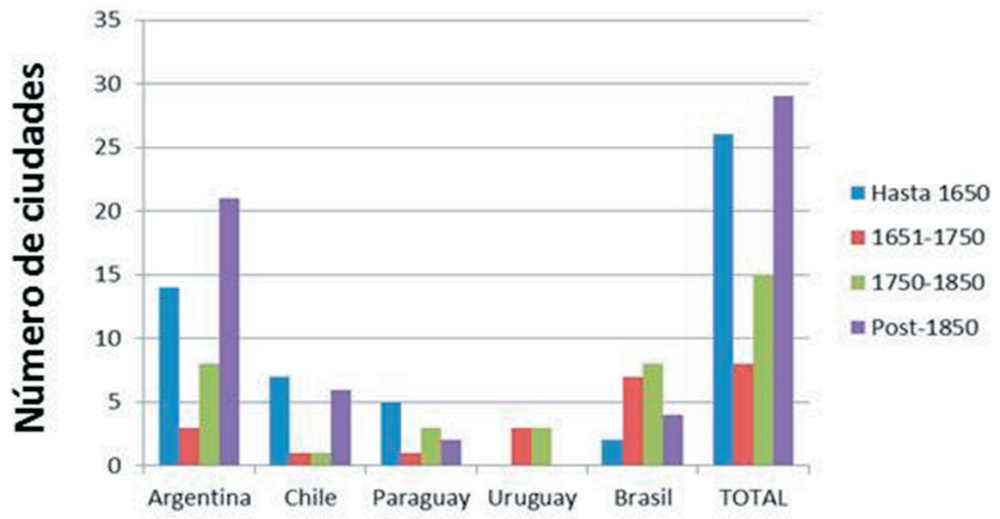

(b)

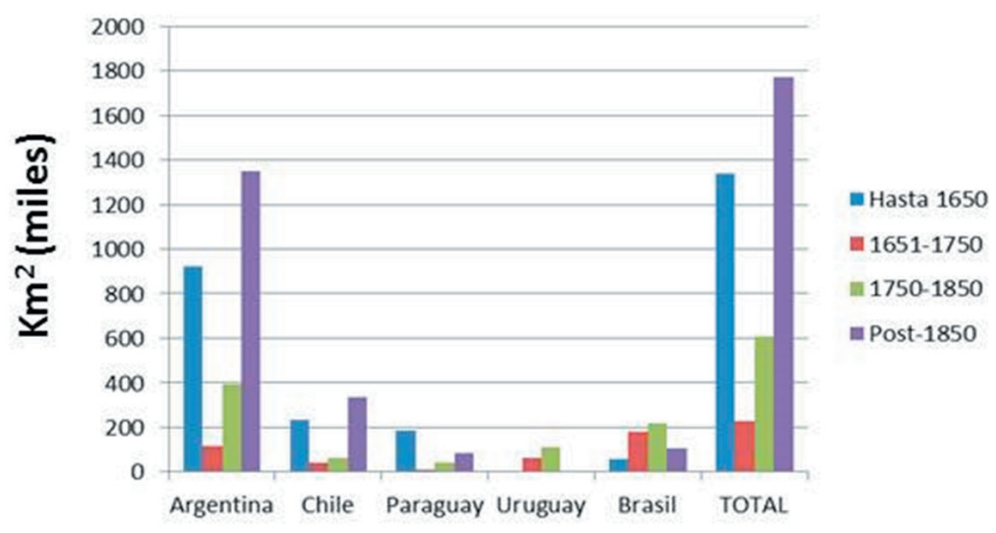

Figura 1. (a) Número de ciudades fundadas en cuatro períodos de tiempo por países. (b) Área de influencia urbana según su fecha de fundación.

Figure 1. (a) Number of cities founded in four time periods by country. (b) Area of influence according to foundation date of the cities. 

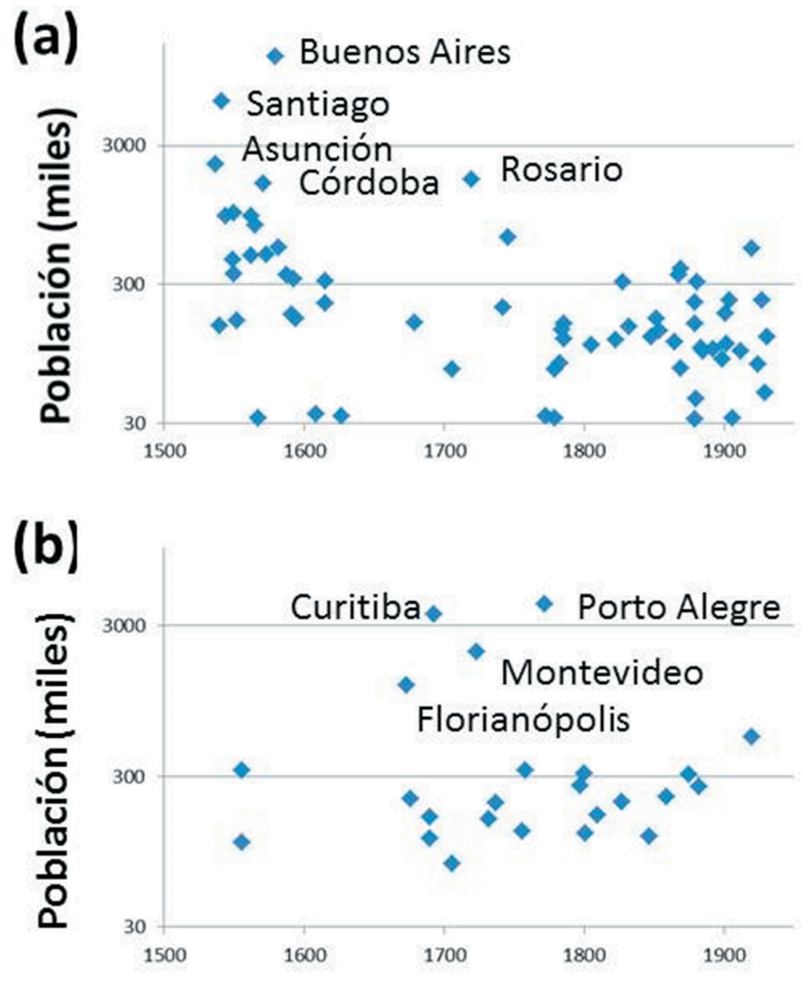

Figura 2. Tamaño poblacional (escala logarítmica) de las ciudades consideradas en este estudio, en relación con la fecha de fundación: (a) en el sector oeste (Chile, Paraguay y Argentina) y (b) en el sector este (Brasil y Uruguay).

Figure 2. Population size (log scale) of the cities considered in this study, in relation to its foundation date: (a) in the western (Chile, Paraguay and Argentine) and (b) eastern (Brazil,

Fecha de fundación Uruguay) sectors.

el sector oeste y mostraron un número alto de fundaciones posteriores a 1700. Si bien aquí también las ciudades con más población son comparativamente antiguas (Curitiba y Florianópolis en el siglo XVII, Porto Alegre y Montevideo en el XVIII), en el caso de Brasil, la fundación de ciudades se prolongó hasta entrado el siglo XX, con ciudades relativamente importantes fundadas hace menos de 100 años, como es el caso de Londrina (Paraná) con más de 500000 habitantes, fundada en 1925.

Los histogramas con área de influencia (Figura 1b) muestran que las áreas bajo influencia de fundaciones recientes (post 1850) incluyen una proporción mayor del territorio. En particular, en Chile y en la Argentina, aunque la población se concentra en ciudades fundadas antes de 1650, la mayor superficie de territorio tiene lugar bajo el área de influencia de ciudades fundadas después de 1850.

\section{Modelado espacial}

El mapa de 'áreas de influencia' o fecha de fundación de la ciudad más cercana, muestra también patrones claros que podrían categorizarse respectivamente como de colonización temprana, tardía, e intermedia. Los sectores de colonización temprana (hasta 1650) incluyen las tres grandes 'rutas' iniciales de colonización española (Figura 3): 1) el centro-norte de Chile, 2) el noroeste $y$ el centro-oeste de la Argentina (en estos dos casos, con las ciudades fundadas por los conquistadores, quienes, partiendo desde Lima, fundaron ciudades en regiones que, en términos generales, se superponían con la avanzada austral del imperio incaico sobre sociedades agro-alfareras preexistentes [e.g., Santiago, Copiapó, Mendoza, San Juan, Santiago del Estero, San Miguel de Tucumán, Córdoba]), y 3) el litoral fluvial de los ríos Paraguay-Paraná-de la Plata, que facilitó la colonización temprana por los conquistadores que ingresaron desde el Atlántico para fundar ciudades como Asunción (Paraguay), Buenos Aires, Santa Fe o Corrientes (Argentina).

Los sectores de colonización tardía (posteriores a 1850) corresponden a zonas donde la población indígena permaneció sin ser dominada por los poderes coloniales hasta el siglo XIX, momento en que los estadosnación independientes (principalmente Chile y la Argentina) las sometieron, estableciendo nuevas ciudades y consolidando los límites políticos que en líneas generales se conservan hasta la actualidad. De estos sectores, el más extenso es la Patagonia argentino-chilena. 


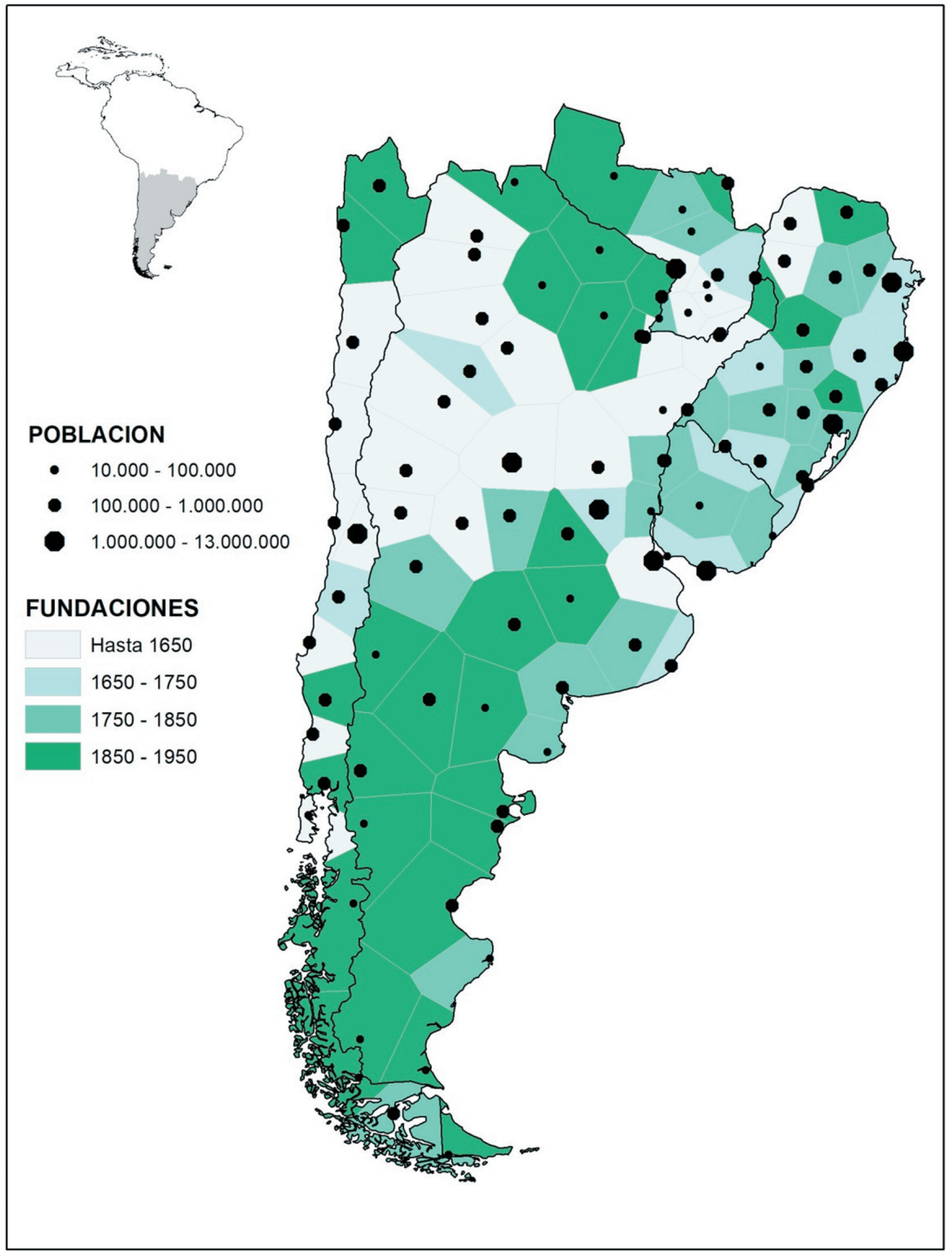

Figura 3. Fechas de fundación de la ciudad 'influyente' más cercana.

Figure 3. Foundation date of the closer 'influencing' city.

También vemos un sector de colonización tardía en los sectores centrales del Chaco Semiárido de la Argentina (oeste de Chaco y Formosa, NE de Santiago del Estero, este de Salta) y Paraguay (departamento Boquerón). En este último, adquiere importancia la colonización por grupos de origen religioso (menonitas) centrado en la ciudad de Filadelfia. También se clasifica bajo este patrón el extremo norte de Chile, bajo la influencia de Antofagasta y Calama, ciudades fundadas en la segunda mitad del siglo XIX en relación a la expansión minera y la guerra del Pacífico o 'del salitre', y algunos núcleos de Brasil, por ejemplo al norte de Rio Grande do Sul

Entre los sectores 'intermedios' se destaca Uruguay, la mayor parte del sur de Brasil, el este de la provincia de Entre Ríos (Argentina) y una zona de 'transición' entre los sectores tempranos y tardíos en el centro sur de la Argentina: área 'de frontera' bajo la influencia de ciudades como Tandil y Bahía Blanca en Buenos Aires, Viedma en Río Negro, Río Cuarto en Córdoba y San Rafael en Mendoza; y sectores en el centro-norte de Paraguay, bajo influencia de poblaciones cercanas al río Paraguay.

\section{Análisis eco-regional}

El análisis discrimina cuatro tipologías de ecorregiones del cono sur de acuerdo a los patrones de edad de la influencia de ciudades, con coherencia geográfica pero menor consistencia en cuanto a características ecológicas (Figura 4). 1) En el noroeste del área de estudio se encuentran las ecorregiones 

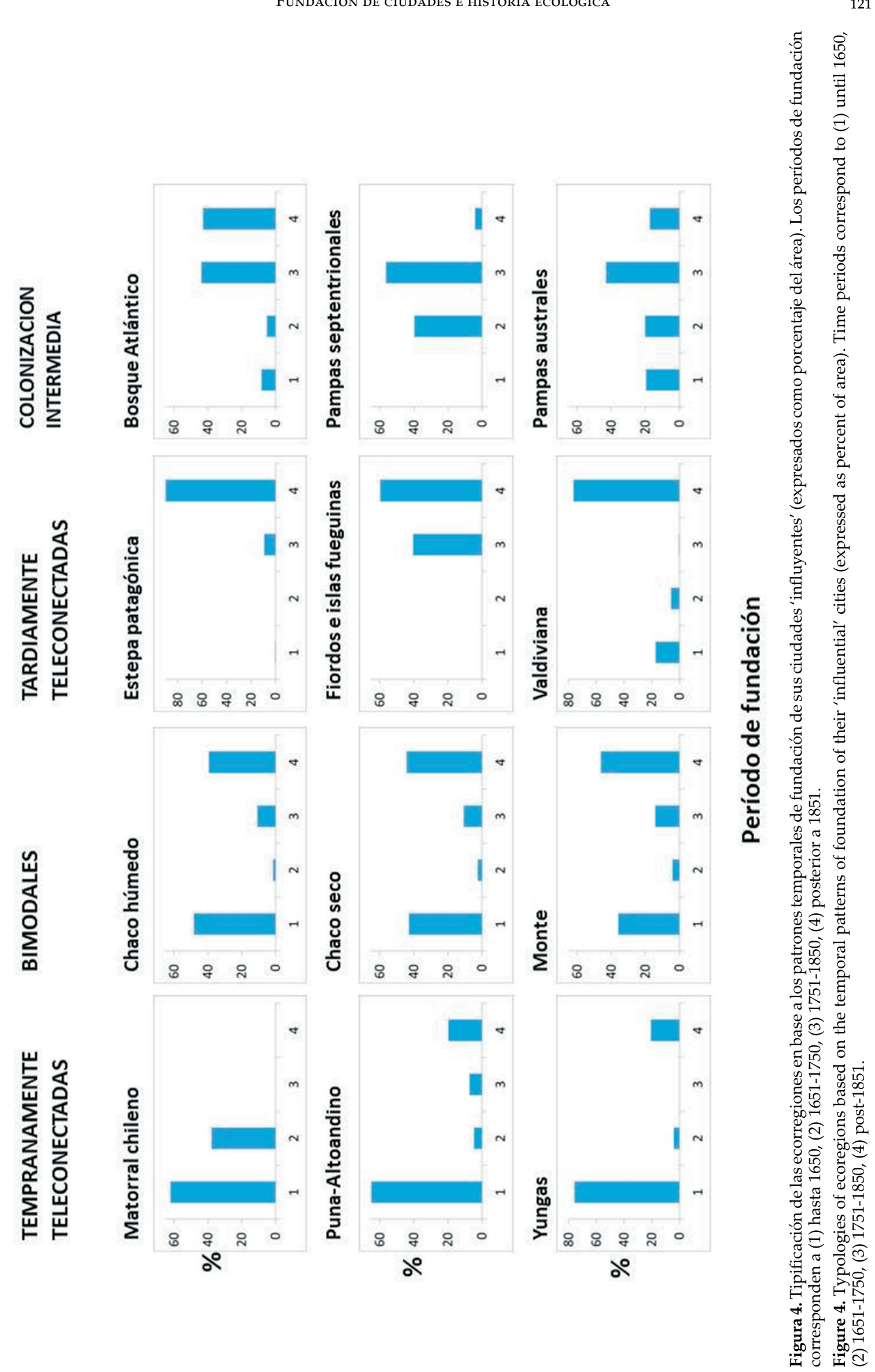

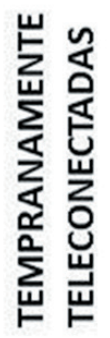
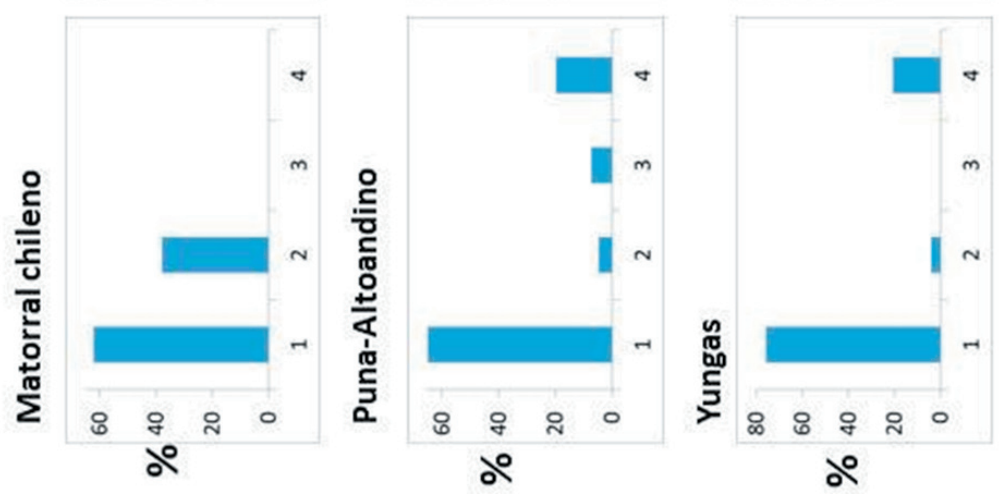
'tempranamente teleconectadas', en las que más de $70 \%$ de su superficie estuvo bajo la influencia de ciudades fundadas antes de 1750, y mayoritariamente durante los primeros cien años de colonización. Esta tipología incluye ecorregiones muy disimiles ecológicamente: el Matorral Chileno (mediterráneo semiárido), la Puna-Altoandina (frío seco) y las Yungas (subtropical húmedo-cálido). 2) Las ecorregiones 'tardíamente teleconectadas' tienen más de tres cuartos de su superficie asociada a ciudades establecidas luego de 1750, y la mayor parte luego de 1850. Incluye las distintas ecorregiones patagónicas frías, tanto boscosas húmedas o como de estepas áridas. En este grupo, sólo los bosques valdivianos presentan algunas ciudades de fundación previa a 1650 (Castro y Valdivia, Chile). 3) Las ecorregiones del noreste con 'colonización intermedia', incluyen los dos tipos de praderas pampeanas y el Bosque Atlántico. Muestran picos de fundación de ciudades en los dos períodos intermedios (entre 1650 y 1850). 4) El Chaco Húmedo, el Chaco Seco y el Monte se congregan en una tipología 'bimodal' en el centro-norte del área de estudio, con fundaciones tanto tempranas como tardías, y menos de $20 \%$ de superficie asociada a fundaciones entre 1650 y 1850.

\section{DiscusióN}

La historia ambiental y socioecológica de las américas experimentó un cambio radical con la colonización europea iniciada a fines del siglo XV y principios del XVI. Una consecuencia clara de ese impacto fue el inicio de un funcionamiento 'teleconectado' con el resto del planeta y una nueva estructura de uso del territorio con creciente impacto de los núcleos urbanos (Mann 2005, 2013; Brailovsky 2006; Williamson 2009; Friss et al. 2015; Grau 2016). Hasta donde sabemos, el presente trabajo es el primer avance en modelar geográficamente el potencial efecto ecológico de este gran evento histórico sobre la socioecología de las américas, en este caso, enfocado en el cono sur del continente.

\section{Aporte metodológico}

Nuestro análisis sugiere que el uso de las fechas de fundación de ciudades representa una herramienta promisoria para estudiar la historia de uso del territorio, ya que permite modelar espacialmente los efectos territoriales de la globalización y los teleacoples asociados por las razones que se exponen a continuación.
1) La creación de ciudades con un evento fundacional discretoy formalmenteestablecido es fácilmente accesible y la metodología es extrapolable al resto de América Latina, donde la dinámica de colonización siguió patrones similares. En unos pocos casos, la fecha de fundación de las ciudades no captura el inicio del uso del territorio dominado por los europeos, y en estos casos, se puede datar esta fecha mediante la búsqueda de información histórica adicional. En otras regiones colonizadas más recientemente (Australia, Nueva Zelanda y Norteamérica), el método también podría aplicarse; no así en regiones donde la formación de ciudades es de más larga data con fundaciones informales y procesos de teleconexion más graduales y ocurridos en un pasado más distante (e.g., Europa y Asia). 2) Los modelos espaciales generados capturan procesos bien conocidos de historia de uso del territorio. Por ejemplo, la tardía colonización europea de la Patagonia y el Chaco se refleja claramente en nuestro modelado espacial, al igual que la colonización diferencial entre Brasil/Uruguay, en contraste con Argentina/Paraguay/Chile.

Entre las limitaciones del método se encuentran la poca resolución espacial. Para avanzar en detalles a escala fina se podría recurrir a datar ciudades y poblados más chicos, lo que es menos accesible y demanda un trabajo historiográfico 'artesanal'. También aparecen algunos 'artefactos' del método derivados del supuesto subyacente que no siempre es válido: las ciudades grandes, al correlacionarse con la edad, son un buen reflejo de la fecha de colonización. Este tipo de limitaciones podrían superarse con un análisis historiográfico detallado y laborioso, pero para una escala geográfica extensa y una resolución espacial moderada no representan grandes ganancias con respecto a los modelos aquí usados.

\section{Patrones espaciales: aportes a la caracterización socioecológica del territorio}

El análisis aquí propuesto permite introducir una nueva variable de interés en la caracterización geográfica de los socioecosistemas sudamericanos. Los patrones espacio-temporales de fundación de ciudades y su área de influencia adquieren relevancia cuando permiten observar tipologías diferentes de las basadas en patrones estrictamente ecológicos. En líneas generales, nuestro análisis discrimina claramente tres sectores de 
'historia de uso teleconectado' que no coincide exactamente con las tipologías ecológicas y en consecuencia agrega información ecológicamente relevante y distintiva a éstas. Las zonas de colonización temprana incluyen tanto sectores subtropicales húmedos (Yungas), como zonas de alta montaña (Puna) o zonas subtropicales y templadas áridas o semiáridas (Monte y Matorral Chileno). Las zonas de colonización tardía incluyen tanto bosques como pastizales templado-fríos de la Patagonia, como los bosques semiáridos de la zona más cálida del área de estudio: el Chaco. Las zonas de colonización intermedia del sur de Brasil y Uruguay manifiestan una historia de colonización que discrimina tanto sus bosques subtropicales húmedos de otros situados más al occidente, como los pastizales de la pampa septentrional de los de la pampa austral, todos sometidos a una influencia antrópica europea relativamente más reciente aunque intensa.

El impacto de las ciudades actuales fue claramente reconocido como un causante de la transformación del paisaje (Sinclair 1966; Peet 1969; Seto et al. 2012; Grau 2016). Sin embargo, el impacto histórico de la fecha de fundación de ciudades sobre el funcionamiento ecológico ha sido poco estudiado. Nuestro análisis provee un enfoque metodológico promisorio para esto, y pone en evidencia que la fecha de fundación de ciudades guarda correlación con el tamaño de las mismas. En consecuencia, además del efecto 'histórico' de la fundación de ciudades, éstas tendrían un potencial efecto actual, al ser un condicionante de tamaño de sus poblaciones y su consecuente área de influencia en el presente.

\section{¿En qué influyen las ciudades?}

No es el objetivo principal de este análisis cuantificar en detalle los efectos de la influencia de las ciudades sobre su entorno ecológico, sino resaltar el potencial legado histórico que las urbes ejercieron y ejercen en el ecosistema. Partimos del supuesto de que la proximidad de una porción del territorio a una ciudad recibe una potencial influencia en el funcionamiento socioecológico del territorio, en particular, generando mecanismos de teleacoples del cono sur sudamericano con el resto del mundo. Para ejemplificar las posibles implicancias socioecológicas de nuestra aproximación a la historia del uso del territorio 'teleconectado' discutiremos cuatro patrones temporales de cambios ecológicos que pueden derivar de la fundación de ciudades.

\section{Cambios irreversibles asociados a la fundación urbana: agricultura, herbivoría y comunidades bióticas asociadas}

Aunque algunas de las ciudades (e.g., Copiapó, Calama, Chile) se establecieron sobre asentamientos indígenas precolombinos con actividad agrícola, la etapa colonial representa una clara expansión cuantitativa y cualitativamente diferente de cultivos. Los cereales del viejo mundo (i.e., trigo, cebada, arroz), los frutales de pepita (i.e., manzana, pera) y carozo (i.e., durazno, ciruelos), la vid, la caña de azúcar, los cítricos, son ejemplos de los cultivos que se expandieron a partir de las ciudades coloniales en el territorio. Inclusive cultivos de origen americano como el algodón tuvieron en muchas regiones un impulso adicional como consecuencia de la colonización. Más recientemente, estos frentes de colonización representan la entrada de nuevos cultivos como la soja o las pasturas implantadas.

La introducción del ganado europeo fue quizás más importante territorialmente, sobre todo en épocas tempranas. Típicamente, la fundación de ciudades resultó en la introducción de ganado que en muchos casos se expandió con velocidad (Melville 1999; Piossek Prebisch 2001; Williamson 2009), lo que imprimió cambios ecológicos asociados a este componente socioecológico. A diferencia de los cultivos agrícolas, que requieren de inversiones y de un mercado para expandirse, el ganado se expandió de manera casi independiente de estos factores, tanto como una forma 'barata' de asentar derechos sobre el territorio como mediante el asilvestramiento espontáneo de los herbívoros introducidos. Los efectos del ganado sobre la ecología de las comunidades naturales son muy variables, con importantes diferencias entre ecorregiones (Mazzini et al. 2018), pero en todo caso representan un cambio potencialmente mayor. Nuestro análisis sugiere que, por ejemplo, el Matorral Chileno, las Yungas o el sector norte del Monte en la Argentina, regiones correspondientes a provincias biogeográficas claramente distintas, con una biota característica (Cabrera and Willink 1980), pasaron rápidamente a tener una comunidad de grandes herbívoros que fue gradualmente dominada por ungulados europeos (i.e., 
caprinos, vacunos, ovinos, equinos) durante los siguientes cuatro siglos. La Puna y el sector austral de la Estepa Patagónica tienen similitudes ecológicas y fitogeográficas, pero su historia de pastoreo difiere en más de dos siglos en cuanto a la llegada del ganado europeo como reemplazante de los camélidos nativos, con impactos potencialmente muy importantes en la dinámica de los ecosistemas. Los árboles, organismos estructurales que acumulan el grueso de la biomasa y determinan el hábitat de los ecosistemas boscosos, son candidatos a reflejar esas diferencias. Por ejemplo, los árboles dominantes de los bosques patagónicos (coníferas, Nothofagus spp.) tienen longevidades que suelen exceder los dos siglos (Villalba and Veblen 1997), lo que implica que se establecieron con una comunidad de herbívoros exclusivamente nativa. En contraste, nuestro análisis muestra que los bosques de yungas estuvieron cerca de alguna ciudad colonial desde hace más de cuatro siglos; incluso, es muy posible que los árboles existentes más viejos, establecidos en el siglo XVII (Cedrela lilloi, Juglans australis) (Villalba et al. 1998) lo hayan hecho con una comunidad de herbívoros con fuerte influencia de ungulados europeos. Además del efecto del pastoreo sobre la estructura y la diversidad vegetal, la introducción del ganado puede haber influido en otros aspectos ecológicos importantes; por ejemplo, reduciendo la cantidad de predadores (e.g., felinos) por caza de protección al ganado, operando como mecanismo de reemplazo de los cultivos indígenas (Noli 1998), o alterando los regímenes de fuego por prácticas de manejo tendientes a mejorar el forraje o por remoción de combustible, generando arbustificación como se observó en el caso del Chaco Semiárido (Morello and Saravia Toledo 1959; Grau et al. 2014).

En general, la colonización europea implica un cambio desde una comunidad de grandes herbívoros nativos (camélidos, cérvidos) a una dominada por especies del viejo mundo (exóticos). Sin embargo, análisis recientes sugieren una perspectiva diferente en el caso de los equinos. Miembros de este linaje (géneros Equus e Hippidion) habitaron las américas por millones de años hasta que se extinguieron en forma simultánea con la llegada de los humanos hace poco más de 10000 años. Con esta perspectiva, la introducción y el asilvestramiento de burros en sectores de la Puna y el Monte (Grau et al. 2020) o de caballos en la Pampa o en la
Patagonia (Núñez et al. 2016), derivadas de la 'teleconexion' colonial, podrían interpretarse como casos de pleistocene rewilding, una suerte de restauración ecológica de las condiciones pre-humanas de los ecosistemas (Donlan et al. 2016).

La fundación de ciudades como sustituto de perturbaciones puntuales con efectos ecológicos duraderos

En el caso de la agricultura y la ganadería, el efecto de la fundación de ciudades puede aceptarse como un cambio relativamente irreversible que se 'irradia' desde los núcleos urbanos y que alteró las comunidades bióticas y que continúa haciéndolo en el presente y hacia el futuro. Otro tipo de efecto ecológico es el de procesos relativamente puntuales en el tiempo ocurridos en asociación con la colonización, que a su vez se correlaciona con la fundación de ciudades. Por ejemplo, Veblen et al. (1999) encontraron que en la transición del siglo XIX al XX, en el noroeste de la Patagonia Argentina se observa un aumento de la frecuencia de fuegos, que en buena medida determinan la estructura del bosque y del paisaje actual en el ecotono bosque-estepa. Este aumento en la frecuencia de fuegos se asociaría a los conflictos territoriales entre la colonización argentina y las poblaciones indígenas, y está bien capturado por la fecha de fundación de las ciudades que aquí documentamos como referentes de esa región: Chos Malal (1887), Bariloche (1902), Neuquén (1904) y Esquel (1906).

Otro tipo de procesos que posiblemente tuvo este tipo de dinámica temporal fue la influencia de enfermedades allóctonas sobre las poblaciones indígenas, que causaron colapsos poblacionales $y$, en consecuencia, la recuperación de ecosistemas naturales. Se conoce que las enfermedades importadas por los europeos provocaron una mortalidad elevada en las poblaciones nativas, que tenían una inmunidad limitada. Este proceso, generalmente asociado a la viruela y el sarampión, tuvo como consecuencia la reducción de los fuegos y la deforestación en muchos ecosistemas de Norteamérica, Centroamérica y la Amazonia, llevados a cabo por las poblaciones indígenas, lo que resultó en una masiva recuperación de estos ecosistemas en los siglos XVI y XVII e incluso posibles efectos climáticos globales (Neble and Bird 2008; Goldweijk et al. 2010; Ellis et al. 2020). Si bien esta asociación entre epidemias 
humanas y sus efectos ecosistémicos está poco estudiada en el cono sur (Prieto and Rojas 2013), es sugestivo que eventos de mortalidad de poblaciones indígenas por epidemias de viruela son reportados para Chile central en la década de 1560 (Piossek Prebisch 2002), en la provincia de Chaco, Argentina (Bonatti and Valdez 2010) y los territorios fueguinos a fines del siglo XIX (Casali 2012). En los tres casos, en forma coincidente con los patrones temporales de influencia urbana aquí detectados.

\section{Correlación fecha-tamaño y retardo en el efecto ecológico}

Hay tipos de desarrollo económicos que dependen de la historia de las ciudades y, en parte, de su edad. Un ejemplo de ello es el turismo, especialmente el que tiene base cultural o histórica (Bertocello 2008). Por otra parte, hay efectos ecológicos que si bien no dependen de la fundación temprana de las ciudades, sí dependen de su tamaño, que como documentamos aquí, se correlaciona en parte con la edad (Figura 2). Ciudades grandes, en particular situadas cerca de zonas montañosas sin potencial de agricultura moderna, pueden favorecer la des-intensificación agrícola y la recuperación de ecosistemas al cambiar la economía regional (Grau et al. 2008; Grau 2016). Ese efecto de las grandes ciudades de favorecer transiciones ecológicas también puede aumentar el potencial de invasión de especies exóticas que generan las ciudades.

La riqueza de especies exóticas y la densidad poblacional están correlacionadas de forma positiva y, en muchos casos, favorece la invasión de especies exóticas (Schwartz et al. 2006; Botham et al. 2009). Montti et al. (2017) analizaron un ejemplo en el cono sur que relaciona las tres variables: documentaron que los principales núcleos de invasión de Ligustrum lucidum, especie invasora originaria de China, ocurren en las cuatro mayores ciudades pedemontanas del centro-oeste y noroeste argentino: Córdoba, San Miguel de Tucumán, Salta y San Salvador de Jujuy. La invasión de Ligustrum aparentemente se inició a fines del siglo XIX; sin embargo, la asociación con grandes ciudades sugiere que la gran masa urbana, vinculada con grandes cantidades de forestación urbana y jardines en los que se usa la especie, debe haber jugado un rol clave. Consistentemente, tanto en Santa Catarina (Baptista and Rudel 2008) como en Uruguay (Haretche and Brazeiro 2017) y en la provincia de Buenos Aires (Ribichich and Protomastro 1998), las tres ciudades más grandes y antiguas también son las que muestran altos niveles de invasión de ligustro en la periferia: Florianópolis, Montevideo y Buenos Aires, respectivamente.

\section{Nichos de colonización tardía reflejados en la historia de ciudades}

La colonización de determinadas áreas del territorio implica un efecto directo sobre ellas. Además, la no-colonización provee oportunidades para que otras formas del uso del territorio -inexistentes en épocas tempranas, pero desarrolladas luego en la historia - aprovechenestos'nichos' geográficoecológicos 'vacíos' para imponer su novedosa impronta en el territorio. Un ejemplo de esto, capturado en este análisis, es la colonización tardía del oeste de Paraguay (departamento Boquerón, con fecha de fundación de su ciudad de referencia, Filadelfia, en 1931). Al no haber tenido colonización convencional por los gobiernos y culturas nacionales hasta inicios del siglo $X X$, este sector del territorio permitió la colonización tardía de grupos menonitas que hoy dominan el uso del territorio. Al igual que en otros países como Bolivia (Killeen et al. 2008), este grupo social tiene características culturales y demográficas muy particulares y genera patrones de uso del territorio y conexiones internacionales muy característicos (Vázquez 2005), claramente diferente del uso del territorio del Chaco Semiárido en la Argentina. Por ejemplo, estos patrones resultaron en combinaciones de manejo del ganado, reservas de agua y remanentes de bosques apropiados para la conservación de grandes mamíferos (Weiler et al. 2020), en simultáneo con una deforestación acelerada y una tendencia al fuerte crecimiento poblacional que, a diferencia de otros sectores agropecuarios, no resulta en emigración a las ciudades. En el área de estudio de este trabajo, patrones similares de uso de nichos territoriales tardíos se podrían observar a escalas más finas en el Chaco Semiárido argentino, también colonizado por menonitas (noreste de Santiago del Estero), galeses en la provincia de Chubut (Argentina) o al norte del estado de Rio Grande do Sul (Trento 1989), donde colonos italianos se establecieron recién a fines del siglo XIX en la zona de influencia de Caixas do Sul (fecha de fundación: 1880). Estos ejemplos ilustran que los patrones culturales y sus vínculos distantes son un componente central del uso del territorio y sus consecuencias ecológicas, lo que a su vez 
se expresa geográficamente en los análisis generados por nuestro estudio.

\section{Conclusiones}

Las ciudades tuvieron y tienen un efecto importante sobre el territorio. No sólo por su impacto directo sino por los 'teleacoples' que nuclean, promueven y regulan. La fecha de fundación de ciudades del cono sur sudamericano es una herramienta promisoria y posiblemente única para contribuir a modelar espacialmente la historia de uso del territorio en las américas 'teleconectado' con el resto del mundo. Estos esfuerzos podrían sumarse a los modelados basados en estudios arqueológico que describen los patrones precoloniales, a fin de lograr una interpretación integral de la historia ambiental y del efecto relativo de este evento único: la colonización europea del continente.

Elenfoqueaquípresentadopermiteidentificar patrones geográficos claros, con potenciales influencias ecológicas y socioeconómicas clave.
En el cono sur, separa claramente la historia de uso post-colonial de la Patagonia y sectores del Chaco (colonizados sobre todo desde fines del siglo XIX), de la del noroeste argentino, centro norte de Chile y ribera del Paraná (colonizado durante el siglo XVI) y del este del área de estudio (pampas y Bosque Atlántico), con una colonización intensa en fechas intermedias. Esta caracterización es un primer avance para explorar patrones territoriales de procesos ecológicos importantes asociados a conexiones socioecológicas distantes ocurridas en distintos tiempos históricos, como cambios en los patrones de agricultura, herbivoría, disturbios, invasión por especies exóticas o tipos de uso del territorio con bases en formas de tenencia y patrones culturales particulares.

Agradecimientos. Este estudio es parte de los proyectos PICT 2018-03881, PICT 2016-2173 y PUE 2016-IER. Lia Montti, Ezequiel Aráoz, Germán Baldi y dos revisores anónimos realizaron críticas enriquecedoras a versiones tempranas del artículo.

\section{REFERENCIAS}

Baptista, S., and T. K. Rudel. 2008. A re-emerging Atlantic forest? Urbanization, industrialization and the forest transition in Santa Catarina, southern Brazil. Environmental Conservation 33:195-202. https://doi.org/10.1017/ S0376892906003134.

Barnosky, A. D., E. A. Hadly, P. González, J. Head, P. David Polly, et al. 2017. Merging paleobiology with conservation biology to guide the future of terrestrial ecosystems. Science 355(6325):eaah4787. https://doi.org/10.1126/ science.aah4787.

Bertocello, R. (ed.). 2008. Turismo y Geografía: Lugares y Patrimonio Natural-Cultural de la Argentina. Ciccus, Buenos Aires. Pp. 272.

Bonatti, A., and J. Valdez. 2012. Historias desconocidas de la Argentina indígena. Edhasa, Buenos Aires.

Botham, M. S., P. Rothery, P. E. Hulme, M. O. Mill, C. D. Preston, and D. B. Roy. 2009. Do urban areas act as foci for the spread of alien plant species? An assessment of temporal trends in the UK. Diversity and Distributions 15:338-354. https://doi.org/10.1111/j.1472-4642.2008.00539.x.

Brailovsky, A. E. 2006. Historia ecológica de Iberoamérica. Ediciones Kraicon, Buenos Aires.

Cabrera, A. L., and A. Willink. 1980. Biogeografía de América Latina. Organización de Estados Americanos. Washington D.C.

Casali, R. 2012. Contacto interétnico en el norte de Tierra del Fuego: la misión salesiana La Candelaria (Río Grande) y la población Selk'nam (1895-1931). Nuevo Mundo Mundos Nuevos. https://doi.org/10.4000/nuevomundo.64311.

Coni, E. A. 1930. Historia de las vaquerías de Río de la Plata (1550-1750). Sección Americana. Boletín de la Real Academia de la Historia 96:262-357.

Crosby, A. 1993. Imperialismo Ecológico: A Expansao Biológica da Europa: 900-1900. Companhia das Letras, Sao Paulo, Brasil. Pp. 319.

Donlan, C. J., J. Bergre, C. E. Bock, J. H. Bock, D. A. Burney, J. A. Estes, D. Foreman, P. S. Martin, G. W. Roemer, F. Smith, M. E. Soule, and H. W. Greene. 2016. Pleistocene rewilding: an optimistic agenda for twenty first century conservation. American Naturalist 168:660-681. https://doi.org/10.1086/508027.

Ellis, E. C., A. H. W. Beusen, and K. K. Golddewijk. 2020. Anthropogenic biomes: 10,000 to 2015 CE. Land 9(5):129. https://doi.org/10.3390/land9050129.

Ellis, E. C., J. O. Kaplan, D. Q. Fuller, S. Vavrus, K. K. Godewijk, and P. Verburg. 2013. Used planet: a global history. Proceedings of the National Academy of Science, USA 110:7978-7985. https://doi.org/10.1073/pnas.1217241110.

Ellis, E. C., and N. Ramankutty. 2008. Putting people in the map: anthropogenic biomes of the world. Frontiers in Ecology and the Environment 6:439-447. https://doi.org/10.1890/070062.

Friis, C., J. O. Nielsen, I. Otero, H. Haberl, J. Niewohner, and P. Hostert. 2015. From teleconnections to telecouplings: taking stock of an emerging framework in land system science. Journal of Land Use Science 11:131-153. https: 
//doi.org/10.1080/1747423X.2015.1096423.

Goldweijk, K. K., A. Beusen, and M. van Drecht. 2010. The HYDE 3.1 spatially explicit data-base of human induced global land use change over the past 12,000 years. Global Ecology and Biogeography 20:73-86. https://doi.org/10.1111/ j.1466-8238.2010.00587.x.

Grau, H. R. 2016. Equilibrios alternativos mediados por decisiones humanas: controles de la estabilidad y eficiencia del uso del territorio y cobertura del territorio en América Latina. Pp. 171-192 en J. S. Postigo and K. R. Young (eds.). Naturaleza y Sociedad. Perspectivas Socio-ecológicas sobre Cambios Globales en América Latina. Instituto de Estudios Peruanos, Lima, Perú.

Grau, H. R., and T. M. Aide. 2008. Globalization and land-use transitions in Latin America. Ecology and Society 13(2): 16. https://doi.org/10.5751/ES-02559-130216.

Grau, H. R., E. Aráoz, C. Navarro, S. A. Nanni, and A. Malizia. 2020. Pathways of megaheribivore rewilding transitions: typologies from an Andean gradient. Elementa: Science of the Anthropocene 8:19. https://doi.org/ 10.1525/elementa.415.

Grau, H. R., M. E. Hernández, J. Gutiérrez, N. I. Gasparri, M. C. Casavecchia, E. Flores-Ivaldi, and L. Paolini. 2008. A peri-urban neotropical forest transition and its consequences for environmental services. Ecology and Society 13:35. https://doi.org/10.5751/ES-02434-130135.

Grau, H. R., R. Torres, N. I. Gasparri, P. G. Blendinger, S. Marinaro, and L. Macchi. 2014. Natural grasslands in the Chaco. A neglected ecosystem under threat by agriculture expansion and forest-oriented conservation policies. Journal of Arid Environments 123:40-46. https://doi.org/10.1016/j.jaridenv.2014.12.006.

Haretche, F., and A. Brazeiro. 2017. Evaluación de métodos de control de Ligustrum lucidum W.T. Aiton adultos en un bosque nativo de Barrancas (Melilla, Montevideo). Pp. 43-45 en Libro de Resúmenes del seminario "Recientes avances para la gestión y conservación del bosque nativo de Uruguay". Ministerio de Agricultura, Ganadería y Pesca, Uruguay.

Killeen, T., A. Guerra, M. Calzada, L. Correa, V. Calderón, M. Soria, B. Quezada, and M. C. Steininger. 2008. Total historical land use change in Eastern Bolivia: who, when, where and how much? Ecology and Society 13(1):36. https: //doi.org/10.5751/ES-02453-130136.

Lambin, E. C., and P. Meyfroidt. 2010. Land use transitions: socio-ecological feedback versus socio-economic change. Land Use Policy 27:108-118. https://doi.org/10.1016/j.landusepol.2009.09.003.

Mann, C. 2005. 1491, New revelations of the Americas before Columbus. Knopf. Nueva York.

Mann, C. 2013. 1493. Uncovering the new world Columbus created. Vintage Books. Nueva York.

Mazzini, F., M. A. Relva, and L. R. Malizia. 2018. Impacts of domestic cattle on forest and woody ecosystems in southern South America. Plant Ecology 219:913-925. https://doi.org/10.1007/s11258-018-0846-y.

Melville, E. 1999. Plaga de Ovejas: Consecuencias Ambientales de la Conquista de México. Fondo de Cultura Económica, México D.F. Pp. 229.

Montti, L., V. Piriz-Carrillo, J. Gutiérrez Angonese, N. I. Gasparri, R. Aragón, and H. R. Grau. 2017. The role of bioclimatic features, landscape configuration and historical land use in the invasion of an Asian tree in subtropical Argentina. Landscape Ecology 32:2167-2185. https://doi.org/10.1007/s10980-017-0563-2.

Morello, J., and C. Saravia-Toledo. 1959. El bosque chaqueño I. Paisaje primitivo, paisaje natural y paisaje cultural en el oriente de Salta. Revista Agronómica del Noroeste Argentino 3:5-81.

Nevle, R, and D. K. Bird. 2008. Effects of syn-pandemic fire reduction and reforestation in the tropical Americas on atmospheric CO2 during European conquest. Paleogeography, Paleoclimatology, Paleoecology 164:25-38. https: //doi.org/10.1016/j.palaeo.2008.03.008.

Noli, E. S. 1998. Algarrobo, maíz y vacas. Los pueblos indios de San Miguel de Tucumán y la introducción del ganado europeo (1600-1630). Mundo de Antes 1:31-67.

Núñez, C. M. V., A. Scorolli, L. Lagos, D. Berman, and A. J. Kane. 2016. Management of free roaming horses. Pp 133-147 en J. I. Ramson and P. Kaszensky (eds.). Wild equids. Ecology, Management, Conservation. John Hopkins University Press.

Peet, J. R. 1969. The spatial expansion of commercial agriculture in the nineteen century: a von Thunen interpretation. Economic Geography 45:283-301. https://doi.org/10.2307/142666.

Piossek Prebisch, T. 2001. Poblar un pueblo. El comienzo del poblamiento de Argentina en 1550. Edición propia. San Miguel de Tucumán. Pp. 516.

Prieto, M. R., and F. Rojas. 2013. Climate Anomalies and epidemics in South America at the end of the Colonial Period. Climate Change 118:641-658. https://doi.org/10.1007/s10584-013-0696-5.

Ribichich, A. M., and J. Protomastro. 1998. Woody vegetation structure of xeric forest stands under different edaphic site conditions and disturbance histories in the Biosphere Reserve 'Parque Costero del Sur', Argentina. Plant Ecology 139:189-201. https://doi.org/10.1023/A:1009718819857.

Seto, K. C., B. Guneralp, and N. L. Hutyra. 2012. Global forecast of urban expansion to 2030 and direct impacts on biodiversity and carbon pools. Proceedings of the National Academy of Sciences, USA 109:16083-16088. https: //doi.org/10.1073/pnas.1211658109.

Sinclair, R. 1966. Von Thunen and urban spraul. Annals of the Association of American Geographers 57:72-87. https: //doi.org/10.1111/j.1467-8306.1967.tb00591.x.

Schwartz, M. W. 2006. Biotic homogeneization of the California flora in urban and urbanizing regions. Biological Conservation 127:282-291. https://doi.org/10.1016/j.biocon.2005.05.017. 
Trento, A. 1989. Do outro lado do Atlántico: um seculo de imigracao italiana no Brasil. Livraria Novel. Sao Pablo. Brasil.

Vázquez, F. 2005. Las reconfiguraciones territoriales del Chaco paraguayo: entre el espacio nacional y el espacio mundial. Revista Académica de Economía 16(28):68-82.

Veblen, T. T., T. Kitzberger, R. Villalba, and J. Donnegan. 1999. Fire history in northern Patagonia: the roles of humans and climatic variation. Ecological Monographs 69:47-67. https://doi.org/10.1890/0012-9615(1999)069[0047: FHINPT]2.0.CO;2.

Verburg, P. H., N. Crossman, E. C. Ellis, A. Heinnimann, P. Hostert, O. Mertz, N. Nagendra, T. Sikor, K. H. Erb, N. Golubiewski, H. R. Grau, M. Grove, S. Konate, P. Meyfroidt, D. Parker, R. R. Chawdhury, H. Shibata, A. Thomson, and L. Zhen. 2015. Land system science and sustainable development of the earth system: a global land project perspective. Anthropocene 12:29-41. https://doi.org/10.1016/j.ancene.2015.09.004.

Villalba, R., H. R. Grau, J. Boninsegna, G. Jacobby, and A. Ripalta. 1998. Tree ring evidence for long term precipitation changes in subtropical South America. International Journal of Climatology 18:1463-1478. https://doi.org/10.1002/ (SICI)1097-0088(19981115)18:13\%3C1463::AID-JOC324\%3E3.0.CO;2-A.

Villalba, R., and T. T. Veblen. 1997. Regional patterns of tree population age structures in northern Patagonia: climatic and disturbance influences. Journal of Ecology 85:113-124. https://doi.org/10.2307/2960643.

Weiler, A., K. Núñez, and F. Silla. 2020. Forest matters: use of water reservoirs by mammal communities in cattle ranch landscapes in the Paraguayan dry Chaco. Global Ecology and Conservation 23:e01103. https://doi.org/10.1016/ j.gecco.2020.e01103.

Williamson, E. 2009. The Penguin History of Latin America. Penguin, Londres. 